Extended Abstract

\title{
Big data in health issues - Perspectives of Omics-Medicine
}

Hans-Werner Mewes ${ }^{1,2}$

${ }^{1}$ Technische Universität München, Lehrstuhl f. Genomorientierte Bioinformatik, Maximus-vonImhof-Forum 3, 85354 Freising-Weihenstephan

${ }^{2}$ Helmholtz Zentrum München, Inst. f. Bioinformatics and Systems Biology, Ingolstädter Landstrasse 1, 85764 Neuherberg

E-Mails: w.mewes@wzw.tum.de

Tel.: +49-89-3187-3581

Accepted:

\section{Introduction}

For the better and the worse, data govern our life. Information and communication, primarily based on language make us human and give us an unbeatable advantage as a species. The digitalized world introduced a technology that changed the value of information fundamentally. Huge data storage are Pandora's box and treasure chests combined. Information is accessible everywhere, easily to be spied but free opinions cannot be suppressed. Health information these days comes with a similar Janus face: huge data amounts generated by new and sophisticated technologies will enable for preventive instead of therapeutic medicine and are becoming individualized. The perspectives and the technological consequences of a data driven medicine need to be reflected. The future of medicine is already a battlefield for its commercial exploration of the health care industry (1). The potential to diagnose, predict, and prevent rare and common diseases is huge. How we will use health information is a social, political, and economic issue of enormous relevance.

\section{Results and Discussion}

Life itself has a disappointing consequence, birth implies disease and death. Medicine fights diseases based on experience and the observation of the individual phenotype such as fever, pain, and blood pressure. With the genetic information at hand, we now can gain insight into the genetic disposition, with the recordings from various "Omics"-technologies. We can monitor the state of an 
individual biological system including its genome. With the medical record available, we can correlate the genetic disposition, the influence of environment and life style, and the medical history of the patient to the disease state. We can monitor success of therapy and evaluate its economic efficiency. The detailed analysis of the benefit of a therapy enables rationale decisions to guide the health care system. The "one fits all" therapeutic classification will be replaced by personalized, data driven, rationale decisions, even down to the individual genetic level, also coined as precision medicine.

The biomedical literature comprises millions of publications every year. For the individual scientist, to stay abreast of one's own field has become impossible. Omics-technologies record a very large number of parameters and conditions. To build and structure biological information systematically and to identify causes for the onset and progress of diseases is more challenging than trying to solve a very large puzzle (2). To deal with data spaces of many thousands of parameters, to cope with the dimensions of time, space and history in biological data means to solve complex problems and to reflect novel concepts to distinguish the solvable from the unsolvable problems.

The new world of a better, more efficient, and precise treatment arrives with huge promises for a better, healthier life. Not unlike the promise of endless growth and wealth, there are obstacles to be jumped over: given the data, can we make the right prediction for the outcome of a treatment? This question comes with an even more fundamental one: is life in any way predictable? Given the information of all parameters gathered from the patient, what can be done for the better of the patient and the society?

\section{Conclusions}

In my talk, I will address some basic questions on disease mechanisms and the "homo informaticus" as the subject - the scientist - and object - the patient - of data driven medicine.

\section{Acknowledgments}

I like to acknowledge Felix Tretter, Thomas Werner and Dan Rujescu for many stimulating discussions on the conditions how genes and the environment interact and shape our body and mind.

\section{References and Notes}

1. Krol, A.; J. Craig Venter's Latest Venture Has Ambitions Across Human Lifespan. Bio.IT World, March 4, 2014

2. Mewes, H.-W.; Perspectives of a systems biology of the brain: The big data conundrum understanding psychiatric diseases. Pharmacopsychiatry, 2013, 46, S2-S9 (2013)

(C) 2015 by the authors; licensee MDPI and ISIS. This abstract is distributed under the terms and conditions of the Creative Commons Attribution license. 